\title{
Use of Self-Calibration Data for Multifunctional MEMS Sensor Prognostics
}

\author{
H. Khan ${ }^{1}$, I. Tahir ${ }^{2}$, A. Richardson ${ }^{1}$
}

\begin{abstract}
This paper proposes a solution to monitor the degradation of a multifunctional MEMS sensor (MFS) and to recalibrate the sensor output accordingly. The solution is able to predict the remaining useful life based on the recalibration history. The MFS used is a dual pressure-humidity hybrid sensor where model data has been used to demonstrate the applicability and the performance of the proposed method for diagnosis, self-correction and prognosis.
\end{abstract}

Index Terms-MEMS, Prognostics, Self-Test, Diagnostics, Reconfiguration, Fault Tolerance, Pressure, Humidity, HUMS

\section{INTRODUCTION}

$\mathrm{T}$ he integration of Health and Usage Monitoring Systems (HUMS) in engineering structures, equipment and devices is of great interest to deliver the required monitoring functions needed for high reliability, availability and safety. Existing applications include safety critical systems including surface terrain vehicles, aviation vehicles, submarines, ships and complex structures $[1,2]$.

The growth in the use HUMS has been a key driver for the sensor market [3]. Recent advances in the complexity, reliability, power efficiency and low development costs of MEMS sensors are of great interest to the HUMS community [4-6].

Sensors are considered to be the single most important component of HUMS, regardless of the usage requirements or application scope [7] as they are key to the monitoring capability of the HUMS framework. The specifications for these health and usage monitoring sensors is however challenging as sensor malfunction or failure can result in situations where the HUMS would not outlast the principle device it is supposed to monitor [8].

Two of the most prevalent sensing capabilities that the vast majority of HUMS require are humidity and pressure sensing. Humidity and pressure are often used in similar environments and even in paired configurations $[5,9,10]$. Considering this, the design of a multifunctional sensor (MFS) that integrates the sensing of these two parameters has significant market potential.

Prognostics delivers the capacity to predict the future health and the remaining useful life (RUL) of a system however, embedded prognostics does not feature in today's commercial MEMS devices. Solutions to deliver this capability are of interest as the integrity of the data generated by MEMS based devices is critical in many applications, especially HUMS. This is the gap this research is seeking to bridge.

This research presented in this paper aims to realize a low

\footnotetext{
${ }^{1}$ Haroon Khan and Andrew Richardson are members of the Department of Engineering, Lancaster University, Lancaster, UK. (h.khan@lancaster.ac.uk and A.Richardson@lancaster.ac.uk).
}

cost prognostics solution for a MEMS based MFS able to measure pressure and humidity concurrently. The design is based on the architecture described in [11]. The work demonstrates that it is feasible to integrate a "lightweight" module with conservative prognostics capabilities in firmware or hardware into this HUMS based sensor with a very low energy footprint.

\section{MultifunCtional SENSOR (MFS)}

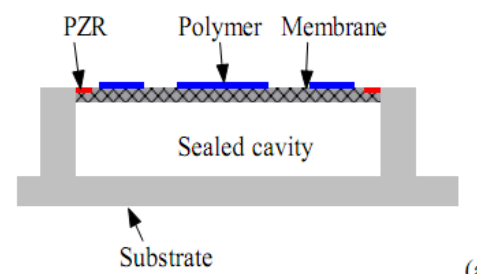

(a)

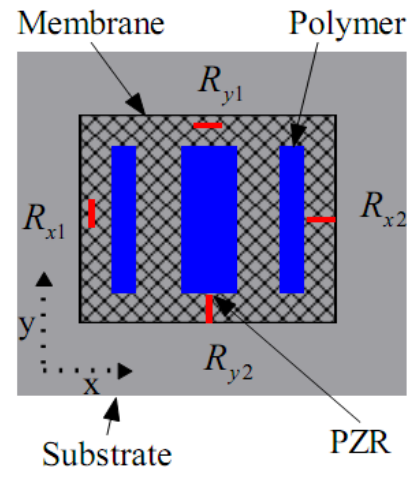

(b)

Fig.1: Top view and cross section of the MFS Schematic [11]

The MFS structure consists of:

- A thin silicon membrane with a very large surface area relative to its thickness.

- Three humidity sensitive polymer beams deposited on the membrane, covering $40 \%$ of the membrane surface.

- Piezoresistors (PZR) embedded into the membrane towards the edges.

An overview of the sensor design is presented in Fig 1.

The membrane is exposed to two sources of mechanical load:

- Load due to atmospheric pressure.

- Load due to humidity induced expansion (and contraction) in the polymer beams.

The strain associated with the mechanical load is most extreme at the periphery $[12,13]$ and hence the piezoresistors [14] are placed in these locations as their resistivity changes

\footnotetext{
${ }^{2}$ Imran Tahir is with Rapiscan Systems Ltd, Stoke-on-Trent, UK (itahir@rapiscansystems.com)
} 
significantly when exposed to high levels of stress at the borders of the membrane [15-17]. Consequently, the physical phenomena most interesting for this research are:

- Piezoresistivity.

- Stress due to Hygroscopic strain (humidity induced strain).

\section{A. Piezoresistivity}

Four piezoresistors are embedded perpendicular and parallel to the edge of the thin film. These resistors are connected in a full bridge configuration where the bridge voltage output is given by (1).

$$
V_{0}=-\frac{\pi_{44}}{2} \cdot \sigma_{p} \cdot V_{i}
$$

Where $\mathrm{V}_{\mathrm{i}}$ is the operating supply voltage to the Wheatstone bridge, $\pi_{44}$ denotes the piezoresistive coefficient of $(100)<100>$ oriented silicon \& $\sigma_{p}$ is the uniaxial stress (in this case, due to pressure). The output of the Wheatstone bridge is a function of one of three piezoresistive coefficients $\left(\pi_{11}, \pi_{12}\right.$ and $\left.\pi_{44}\right)$. These coefficients are functions of doping concentration, type of dopant and temperature $[18,19]$. Of these, $\pi_{44}$ is the most suitable for its ability to mitigate various second order effects

The stress induced on the membrane, in this case due to applied pressure can be expressed by Hooke's Law (2):

$$
\sigma_{p}=\epsilon . E
$$

Where $\epsilon$ is the induced strain and $E$ is the Young's Modulus also known as the modulus of elasticity. Equation (2) can be substituted in (1) to get expression 3 as follows:

$$
V_{0}=-\frac{\pi_{44}}{2} \cdot \epsilon \cdot E \cdot V_{i}
$$

Equation (1) and (3) can be used to calculate the potential difference across the bridge. In the case of the MFS the compressive stress is of interest, where $\sigma_{p}$ is pressure induced stress applied to the sensor.

\section{B. Hygroscopic Strain and the Cumulative Effect}

Due to their sensitivity to humidity, polymer beams are often used as the sensing elements in humidity sensors [20]. Humidity induces changes in volume of a polymer structure due to absorbed moisture [21, 22]. The reversible hygrometric volume expansion due to humidity can be expressed by (4) [22] for a constant temperature:

$$
\epsilon_{H y g}=\alpha_{H y g} \cdot R H
$$

Where RH is the relative humidity, $\alpha_{\mathrm{Hyg}}$ is the linear coefficient of humidity and $\epsilon_{H y g}$ is the hygroscopic strain on the polymer beam. There are three polymer beams deposited on top of the MFS membrane. Absorbed moisture due to humidity exerts mechanical stress [23] $\sigma_{\mathrm{RH}}$ That can be expressed as (5).

$$
\sigma_{\mathrm{RH}}=\left(\alpha_{\text {Hyg }} \cdot \mathrm{RH}\right) . \mathrm{E}
$$

And consequently (2) \& (5) can be used to express the induced strain strain due to relative humidity as shown in (6):

$$
\epsilon_{R H}=\left(\alpha_{H y g} \cdot R H\right)
$$

If there is a piezo resistor embedded in the periphery of the membrane (region of maximum stress), the induced stress would result in a change in potential difference across the full Wheatstone bridge. By applying (6) and (3) for hygroscopic strain, we will arrive to (7):

$$
V_{0}=-\frac{\pi_{44}}{2} \alpha_{H y g} \cdot E \cdot V_{i} \cdot R H
$$

Equation (1) can be reconstituted to accommodate both forms of stress that would affect the voltage across the resistor.

$$
V_{0}=-\frac{\pi_{44}}{2} \cdot V_{i} \cdot\left(\sigma_{p}+\sigma_{R H}\right)
$$

Where the stress due to relative humidity can be replaced with a term consisting of the relative humidity, Young's modulus and the Coefficient of Moisture Expansion (of the polymer beam) as expressed in (9):

$$
V_{0}=-\frac{\pi_{44}}{2} \cdot V_{i} \cdot\left(\sigma_{p}+\alpha_{H y g} E \cdot R H\right)
$$

This is a reasonable expression to present the output of a system as a change in potential difference, where it is a function of the stress due to pressure as well as the humidity itself. This will be used a frame of reference for the validation of the simulation results.

In [10], pressure on the silicon membrane has been directly equated to stress due to pressure. Equation (9) can be used in that context. However, the mathematical representation needs further refinement where there are restrictive boundaries to the transduction of the stress. In such a case, stress cannot be equated to the pressure. A simplified function to derive stress due to pressure in such a situation can be expressed as in [10] in equation (10):

$$
\sigma_{p}=\beta \cdot \frac{p b^{2}}{h^{2}}
$$

Where $p$ is the pressure applied on the silicon membrane, $h$ is the thickness of the thin film membrane, $\beta$ is the dimensional coefficient of due to $\mathrm{a} / \mathrm{b}$ (Table 1$), b$ is the length of the membrane and $a$ is the breadth of the membrane. The stress is presented as a function of not only the pressure but also the structural dimensions of the membrane i.e. the length, breadth and thickness.

\begin{tabular}{lllrrrrr}
\multicolumn{10}{c}{ TABLE 1 } \\
\multicolumn{10}{c}{ Coefficients for maximum stress due to pressure [10] } \\
a/ & 1 & 1.2 & 1.4 & 1.6 & 1.8 & 2 & $\infty$ \\
b & & & & & & & \\
\hline$\beta$ & 0.307 & 0.383 & 0.435 & 0.46 & 0.487 & 0.497 & 0.5000 \\
& 8 & 4 & 6 & 8 & 2 & 4 &
\end{tabular}

The resulting expression to calculate the output of a sensor consolidating both the pressure and humidity for a fixed temperature can be expressed as follows:

$$
V_{0}=-\frac{\pi_{44}}{2} V_{i} \cdot\left(\beta \frac{p b^{2}}{h^{2}}+\alpha_{H y g} E . R H\right)
$$

This expression can be further generalized as follows [24]:

$$
V_{0}=\left(-\frac{\pi_{44}}{2} \cdot V_{i} \cdot\left(\beta \cdot \frac{p b^{2}}{h^{2}}+\alpha_{H y g} \cdot E \cdot R H\right)\right) \cdot \frac{n}{4}
$$

Where $n$ represents the number of active gauges in the Wheatstone bridge i.e. $n=1,2$ or 4 for quarter, half and full bridge respectively. 


\section{Characterization of the MFS Piezoresistive Grid}

Temperature and doping levels in piezoresistors cause the most significant drift in the output characteristics of pressure sensors [25]. Considering the models available [26-28], the Kanda Model [29] has been selected due to the ease with which a good estimate of the piezoresistance coefficient can be computed [15, 28]. This coefficient is dependent on temperature with respect to doping concentrations of the piezoresistors.

1) The Piezoresistive Coefficient

According to Kanda [29, 30], the piezoresistive coefficient is characterized as:

$$
\pi(N, T)=\pi\left(N_{0}, 300 K\right) P(N, T)
$$

Where $\pi\left(N_{0}, 300 K\right)$ is the value of the piezoresistive coefficient for a low doped p-type resistor, $N$ is the doping value, $T$ is the absolute $(\mathrm{K})$ temperature, and $P(N, T)$ is the piezoresistance factor given by:

$$
P(N, T)=\frac{300}{T} \cdot \frac{1}{\delta}
$$

where $\delta=\left[1+\exp \left[-\frac{E_{f}}{K_{b} T}\right]\right] \ln \left[1+\exp \left[-\frac{E_{f}}{K_{b} T}\right]\right]$ with $E_{f}$ being the Fermi Energy in the doped p-type Silicon and $K_{b}$ Boltzmann's constant, The coefficient $\pi_{44}$ used in the mathematical model of the MFS was derived using (13) and (14). Studies have revealed that:

- Increasing doping concentration and/or temperatures respectively cause a lowering in piezoresistive sensitivity [25] and;

- The estimation of the coefficient by Kanda's method is best for doping levels up to $5 \times 10^{19}$ atoms $/ \mathrm{cm}^{3}$.

\section{a) Temperature Effect on piezoresistive response}

The effect of the temperature on the bridge output voltage under a uniform load can be determined using the following relationship [15, 25, 26, 28]:

$$
\frac{\Delta V}{V_{a}}=\frac{\pi_{44}(N, T) \cdot P}{2} \cdot\left(\frac{a}{h}\right)^{2} \cdot\left(\sigma_{x x}-\sigma_{y y}\right)
$$

Where $V_{a}$ is the supply voltage to the bridge, $h$ is the thickness of the membrane, $a$ is the surface area of the membrane and $P$ is the uniform pressure applied on the membrane. A decrease in bridge output voltage is observed with increase in temperature. However, quite importantly, this sensor output response is linear within the operational temperature range.

\section{b) Piezoresistive sensitivity}

The doping concentration of the piezoresistors is the primary variable that affects the sensitivity of piezoresistive sensors. The relationship between doping concentration and sensitivity can be expressed as follows [25, 28]:

$$
S(T, N)=\frac{\pi_{44}(N, T)}{2}\left(\frac{a}{h}\right)^{2}\left(\sigma_{x x}-\sigma_{y y}\right)
$$

It has been observed that both an increase in the thickness of the membrane and increase in doping levels reduces the pressure sensitivity of the piezoresistive elements [25]. It has been found that doping concentration should not exceed $10^{19}$ atoms $/ \mathrm{cm}^{3}$.

\section{SENSOR MODELING}

The first objective in the development of FEM (finite element model) of the MFS model was to validate its behavior. The second objective was to utilise the model to generate a sizeable data set to characterise system behavior and hence develop the prognostics methodology. The final objective was to test the prognostics module by introducing various failures in the model and validate its accuracy and performance.

\section{A. FEM Model}

The Finite Element Analysis (FEA) method has been used to model and measure key parameters of the Multi-functional Sensor (MFS). The MFS was modeled and simulated using COMSOL Multiphysics ${ }^{\circledR}$ 4.4. Details of the FEM model are shown in Table 2. Structural parameters are as follows:

- Piezoresistors: $10 \mu \mathrm{m} \times 50 \mu \mathrm{m} \times 1 \mu \mathrm{m}$ each

- Silicon Membrane: $500 \mu \mathrm{m} \times 500 \mu \mathrm{m} \times 10 \mu \mathrm{m}$ and

- Polymer Beams: $1 \times$ central $400 \mu \mathrm{m} \times 100 \mu \mathrm{m} \times 5 \mu \mathrm{m}$ and

\begin{tabular}{|c|c|c|}
\hline Structure & Modelling Element & Properties \\
\hline Polymer Beams & $\begin{array}{l}\text { Isotropic } \\
\text { Sorption Mechanical } \\
\text { Transduction (study) } \\
\text { Free Triangular Mesh } \\
\text { Minimum Element Size } \\
0.5 \mu \mathrm{m}\end{array}$ & $\begin{array}{l}\text { Young's Modulus }=7.5 \\
\text { GPa } \\
\text { Density }=1 \times 10^{-15}{\mathrm{~kg} \mu \mathrm{m}^{-3}} \\
\text { Poisson's Ratio }=0.35 \\
\text { Humidity Expansion } \\
\text { Coefficient }=1 \times 10^{-4} / \% \mathrm{RH}\end{array}$ \\
\hline $\begin{array}{l}\text { Silicon } \\
\text { Membrane }\end{array}$ & $\begin{array}{l}\text { Isotropic, } \\
\text { Surface } \\
\text { (study), Stress-Strain } \\
\text { Free Triangular Mesh } \\
\text { Minimum Element Size } \\
0.5 \mu \mathrm{m}\end{array}$ & $\begin{array}{l}\text { Young's Modulus }=1381 \\
\mathrm{GPa} \\
\text { Density }=2.33 \times 10^{-15} \mathrm{~kg} . \\
\mu \mathrm{m}^{-3} \\
\text { Poisson's Ratio }=0.278\end{array}$ \\
\hline $\begin{array}{l}\text { Piezoresistors } \\
\text { (p-type) } \\
\text { (Polycrystalline } \\
\text { Crystal) }\end{array}$ & $\begin{array}{l}\text { Anisotropic } \\
\text { Piezoresistivity Boundary } \\
\text { Currents (study), } \\
\text { Free Triangular Mesh } \\
\text { Minimum Element Size } \\
0.1 \mu \mathrm{m}\end{array}$ & $\begin{array}{l}\text { Resistivity }=7.8 \Omega \mathrm{cm} \\
\text { Resistance (under } 0 \\
\text { stress) }=390 \mathrm{k} \Omega\end{array}$ \\
\hline
\end{tabular}
$2 \times$ adjacent $400 \mu \mathrm{m} \times 50 \mu \mathrm{m} \times 5 \mu \mathrm{m}$

$$
\text { TABLE } 2
$$

Parameters of the FEM model used in the simulation

The piezoresistors are composed of silicon with doping levels defined by the piezoresistive coefficient $\pi_{44}$. Piezoresistors parallel and perpendicular to the stress respectively are paired together (as a half bridge). This is the best configuration of the piezoresistors [28] offering highly desirable low thermal sensitivity [28].

The Physics Models employed in COMSOL were "Structural Mechanics $\rightarrow$ Piezoresistivity \& Boundary Currents" and "Structural Mechanics $\rightarrow$ Joule Heating \& Thermal Expansion". Both studies were Stationary. The "Joule Heating \& Thermal Expansion" was used to emulate the deformation of the polymer beams due to moisture absorption, as well as the resulting strain on the membrane. "Piezoresistivity \& Boundary Currents" was used to apply pressure on the membrane surface 
and measure the boundary currents for a fixed potential difference across the 4 individual piezoresistors. All three major physical components were meshed as free triangular elements, with element size varying between $0.5 \mu \mathrm{m}$ and $0.1 \mu \mathrm{m}$. Parametric sweep was used for the static analysis of multiple combinations of applied pressure and humidity within a specified range and intervals to measure the current across the resistors for a fixed voltage (to enable the calculation of the resistance).
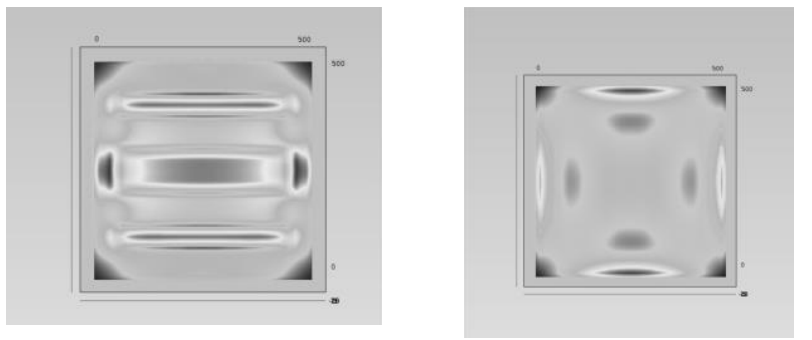

Fig 2: left: The MFS at maximum humidity induced mechanical stress under no pressure and right: under zero percent humidity (i.e. no mechanical stress due to polymer beams)

The sensor was studied for all combinations of pressure: ranging from 0 to $0.2 \mathrm{MPa}$ (2 atmospheres) at intervals of 0.02 $\mathrm{MPa}$ and humidity: ranging from 1 to $100 \%$ at $1 \%$ intervals. Fig 2 shows the sensor at no pressure with maximum humidity and $0 \%$ humidity at maximum pressure respectively.

\section{B. Post FEM Processing}

The two pairs of piezoresistors are realized as two Half Wheatstone Bridges with their voltage outputs $\mathrm{V}_{\mathrm{x}}$ and $\mathrm{V}_{\mathrm{y}}$ respectively (Fig 4) with an input of 5 volts each. Increasing load decreases $R_{\mathrm{y} 1}$ and $\mathrm{R}_{\mathrm{x} 1}$ resistances while increasing $\mathrm{R}_{\mathrm{x} 2}$ and $\mathrm{R}_{\mathrm{y} 2}$.
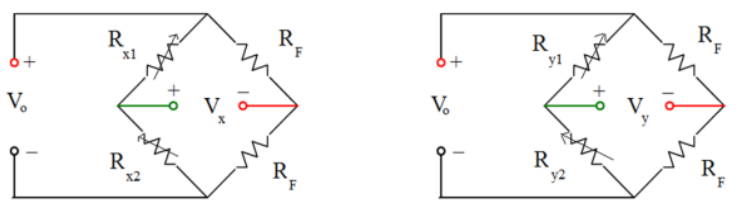

Fig 4: Two Half-Wheatstone bridges

The $\mathrm{V}_{\mathrm{x}}$ and $\mathrm{V}_{\mathrm{y}}$ sensor outputs are processed further within MATLAB to compute the pressure and humidity measurements based on the expression shown in equations (17) \& (18).

$$
\begin{gathered}
p=\frac{\alpha_{\varphi y} \cdot V_{x}-\alpha_{\varphi x} \cdot V_{y}}{\alpha_{\varphi y} \cdot \alpha_{p x}-\alpha_{p y} \cdot \alpha_{\varphi x}} \\
\varphi=\frac{\alpha_{p x} \cdot V_{y}-\alpha_{p y} \cdot V_{x}}{\alpha_{\varphi y} \cdot \alpha_{p x}-\alpha_{p y} \cdot \alpha_{\varphi x}}
\end{gathered}
$$

Where: $V_{x} \& V_{y}$ are the output voltages (from COMSOL forming an input to Spice), $p$ is the pressure, $\varphi$ is humidity, $\alpha_{\varphi y} \& \alpha_{\varphi x}$ are the coefficients of Humidity and $\alpha_{p y} \& \alpha_{p x}$ are the coefficients of Pressure.

For an open chamber paradigm, the starting coefficients of pressure and humidity were recalibrated to the values presented in Table 3:
TABLE 3

Initial Values of the Humidity and Pressure Coefficients

\begin{tabular}{rccc}
\hline $\boldsymbol{\alpha}_{\boldsymbol{p} \boldsymbol{x}}$ & $\boldsymbol{\alpha}_{\boldsymbol{p y}}$ & $\boldsymbol{\alpha}_{\boldsymbol{\varphi x}}$ & $\boldsymbol{\alpha}_{\boldsymbol{\varphi y}}$ \\
\hline-975 volts/Pa & -2368.75 volts/Pa & -0.975 volts/\%RH & -2.256 volts/\%RH
\end{tabular}

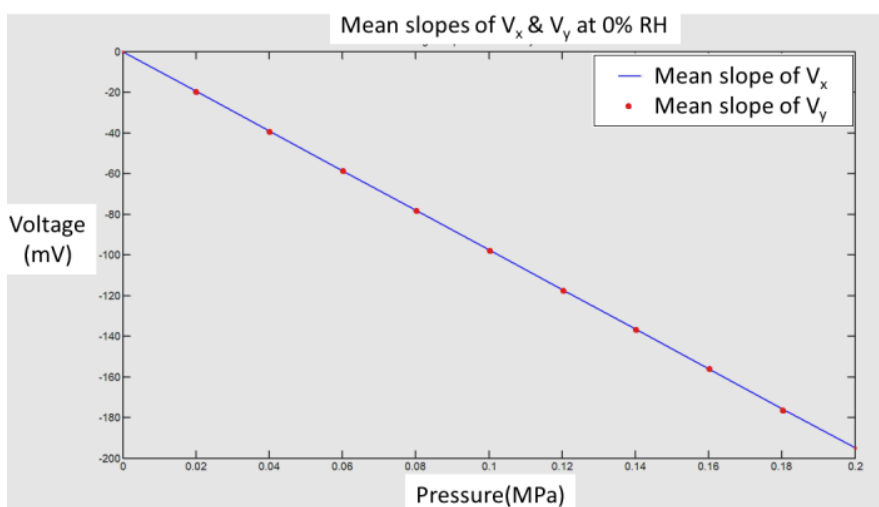

Fig 5: The mean sensitivity slope of the MFS as a function of applied pressure at $0 \%$ humidity. The unit of pressure is $\mathrm{MPa}$

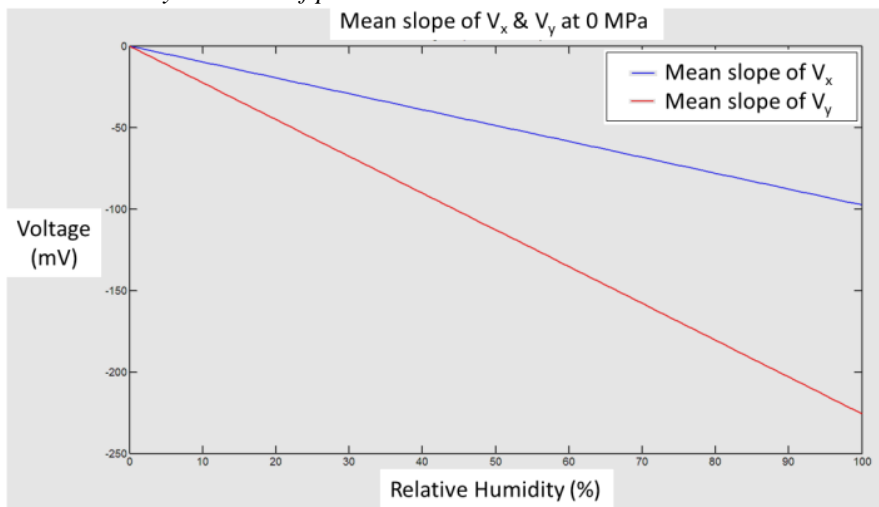

Fig 6: The mean sensitivity slope of the MFS as a function of humidity at OMPa pressure

Fig 5 and 6 show the average sensitivity of the Multifunctional Sensor (MFS) as a function of pressure (at $0 \% \mathrm{RH}$ ) and as a function of humidity (at $0 \mathrm{MPa}$ pressure) respectively. This behavior is consistent for all combinations of pressure and humidity.

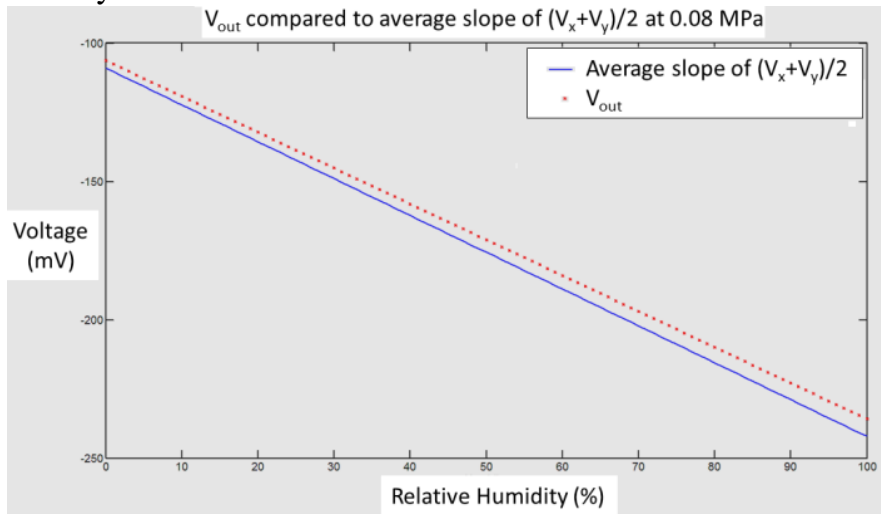

Fig 7: The output $V_{\text {out }}(11)$ in red dots compared to the average slope of (Vy$V x)$ in blue where the $x$-axis is the relative humidity (\%) and the $y$-axis is the voltage $(\mathrm{mV})$

\section{Validation}

Consistent correlation was identified between the output of the MFS and the mathematical model, where a relationship between the average slope of $\mathrm{V}_{\mathrm{y}}-\mathrm{V}_{\mathrm{x}}$ and $\mathrm{V}_{\text {out }}$ as expressed in equation 11 was characterised (Fig 7). 


\section{FAult DeteCtion AND PROGNOSTICS}

The MFS model has been used to simulate normal and faulty modes of operation of the sensor. The resulting data (for faulty sensors) has been used to test and validate the performance of the fault correction and prognostics algorithms

\section{A. Failure Mechanism Simulation}

The most vulnerable part of sensors similar to the MFS is the thin membrane. The standard failure mechanisms associated with such a membrane based structure are due to media incompatibility (stress due to direct interaction with environmental agents), thermal stress and mechanical stress. The main effect of these sources of stress is a change in the elasticity of the membrane. This has been simulated in COMSOL by varying the elasticity of both the silicon membrane and the polymer beams. This has been achieved parametrically by changing the modulus of both the membrane and beams.

\section{B. Operational Overview}

The final sensor is constructed of 4 redundant MFSs fabricated and packaged together on a single substrate. The architecture utilizes this redundancy to facilitate a series of selftests. Consequently, the system has two modes of operation, a Normal Mode of Operation (NMO) and a Test Mode (TMO). The (TMO) provides for additional layers of reliability by supporting:

- Detection of faulty sensors.

- Calibration and alignment of the faulty sensors.

- Health prognostics for each of the individual sensors \&

- An early failure detection window.

\section{Normal Mode of Operation (NMO)}

In normal mode, the raw outputs from the four sensors are digitized and subjected to pre-processing. After pre-processing, the Pressure and Humidity measurements are calculated for each sensor based on their respective coefficients. Any sensor output with an anomalous value for either pressure or humidity is ignored. After data fusion, the system outputs a reading for Pressure and Humidity that are the averages of the valid pressure and humidity outputs produced by the individual sensors, respectively.

\section{Test Mode of Operation (TMO)}

This system has two test modes. The first test mode uses Built-In Self-Test (BIST) for system validation. In case the BIST detects an anomaly in any one of the sensors, auto calibration for self-correction is performed. The second mode performs a prognostic analysis on the system and predicts the remaining useful life (RUL) of the individual sensors as well as the overall system. This provides a high degree of fault tolerance and excellent health management capabilities.

\section{ERRor DETECTION AND FAULT CALIBRATION (FC)}

Fig 8 illustrates the algorithm for the real time calibration of faulty sensors to maintain a correct output. This section will discuss the algorithm in detail.

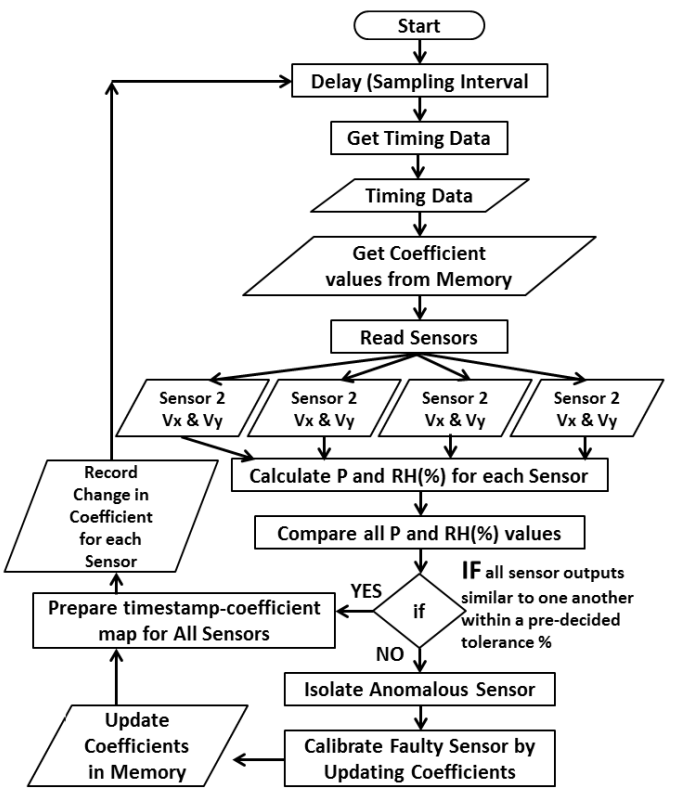

Fig 8: Error Detection and Self Calibration flow chart - The sensor outputs are compared and modify sensors' coefficients for any anomalous sensor that is detected. The values of all coefficients are logged, irrespective of whether they have been changed or not. This forms the input to the prognostics module

The aim of the first program is to detect and isolate faulty sensors and then recalibrate their coefficients to introduce a correcting bias to the output. This program runs a built in selftest at regular periodic intervals. For the purposes of our experiments, the self-test and calibration was run every 10 seconds.

As indicated in the flow chart, at the time of the initiation, the current time stamp is recorded. The testing module then uploads the current values of the coefficients for each sensor. The system maintains 4 reference coefficients for each sensor with initial values as described in Table 3 .

The pressure and humidity output of all 4 sensors are compared, as long as they are similar to one another within a configurable level of tolerance. If an anomalous sensor is detected over the course of the self-test, the algorithm updates the coefficients of that sensor to re-calibrate the output. The recalibration is based on the following criterion:

1. The threshold of deviation is determined by a configurable percentage relative to the correct output derived from the reference sensors, as long as there are at least two sensors that are reasonably consistent $( \pm 0.01 \%)$. If the deviation of the faulty sensor(s) is beyond that threshold, the unit is declared faulty.

2. If at any point, no two sensors outputs are mutually consistent, the unit is declared faulty.

The coefficients due to pressure are calibrated using equations (19) and (20) with the faulty $\mathrm{V}_{\mathrm{x}}$ output while equations (21) and (22) use the faulty $\mathrm{V}_{\mathrm{y}}$ output to recalibrate the humidity coefficients, where " $n$ " is the test iteration ( $n$ being the current and $n-l$ being the previous and so on):

All the coefficients are saved in memory along with the current time stamp. In the case of faulty sensors, the re-calibrated values of the coefficients are stored.

Several hundred tests were conducted to validate the 
performance of this algorithm.

$$
\begin{aligned}
\alpha_{p x_{n}} & =\frac{V_{x_{\text {fault }}} \cdot \alpha_{p x_{n-1}}}{P \cdot \alpha_{p x_{n-1}}+H \cdot \alpha_{\varphi x_{n-1}}} \\
\alpha_{\varphi x_{n}} & =\frac{V_{x_{\text {faulty }} \cdot} \cdot \alpha_{\varphi x_{n-1}}}{P \cdot \alpha_{p x_{n-1}}+H \cdot \alpha_{\varphi x_{n-1}}} \\
\alpha_{p y_{n}}= & \frac{V_{y_{\text {faulty }} \cdot} \cdot \alpha_{p y_{n-1}}}{P \cdot \alpha_{p y_{n-1}}+H \cdot \alpha_{\varphi y_{n-1}}} \\
\alpha_{\varphi y_{n}}= & \frac{V_{y_{f a u l t y}} \cdot \alpha_{\varphi y_{n-1}}}{P \cdot \alpha_{p y_{n-1}}+H \cdot \alpha_{\varphi y_{n-1}}}
\end{aligned}
$$
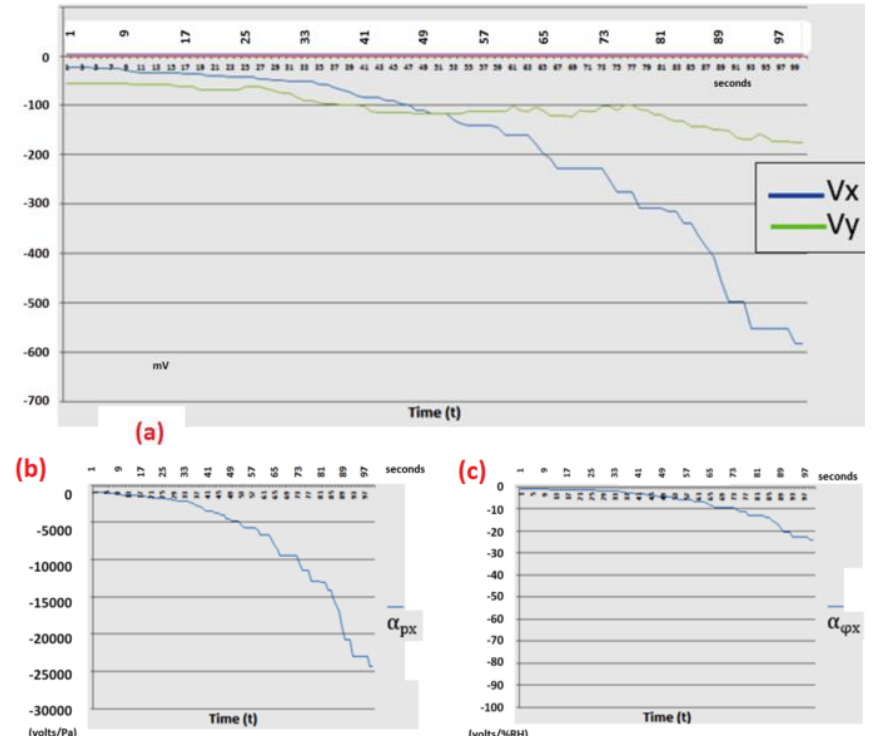

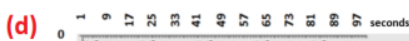
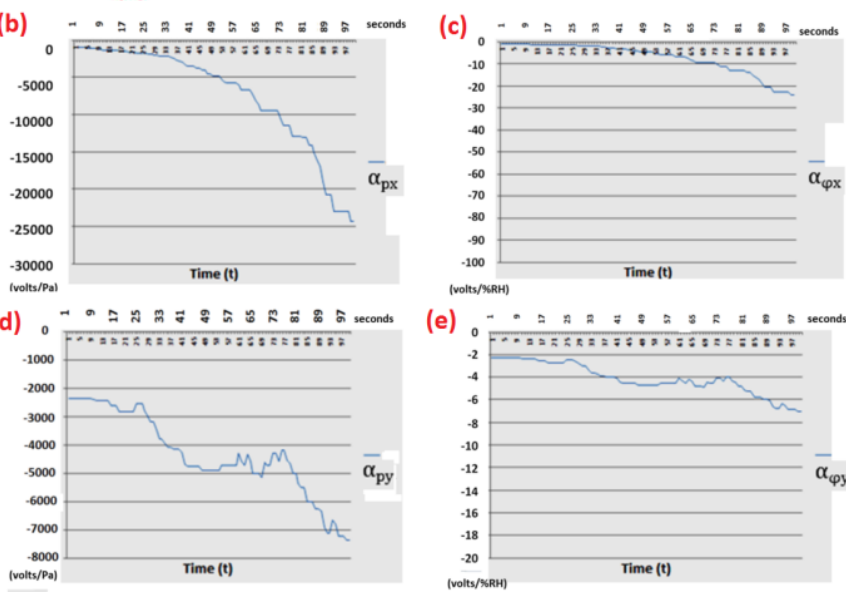

(volts/:SRH)

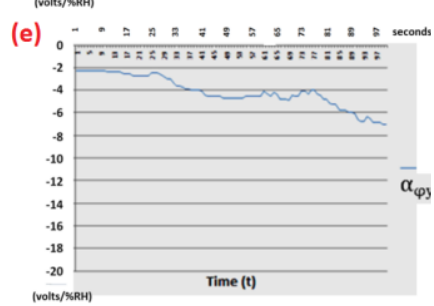

Fig 9 (a) shows the changes in the Vx and Vy outputs of the faulty sensor over time $(b)$ and $(c)$ show the correction in the pressure coefficients while (d) and (e) show the correction in the humidity coefficients for a faulty sensor at $0.02 \mathrm{MPa}$ pressure and $4 \%$ relative humidity.

Fig 9 illustrates one of these tests. The system updates the coefficients following detection of an inconsistent output.

\section{PROGNOSTICS}

The prognostics algorithm uses data generated by the recalibration algorithm discussed in the previous section. Changes in the pressure and humidity coefficients form the input to the prognostics algorithm. A set of test conditions (Table 4) have been applied with Fig's 10 and 11 recording the voltage output $\left(\mathrm{V}_{\mathrm{x}} \& \mathrm{~V}_{\mathrm{y}}\right)$ of healthy and faulty sensors respectively.
TABLE 4

Changing input Humidity and Pressure

\begin{tabular}{ccc}
\hline $\begin{array}{l}\text { Applied Pressure } \\
(\mathrm{MPa})\end{array}$ & $\begin{array}{l}\text { Applied Humidity } \\
(\% \mathrm{RH})\end{array}$ & $\begin{array}{l}\text { Exposure } \\
\text { Time }(\mathrm{sec})\end{array}$ \\
\hline 0.2 & 100 & 5 \\
0.2 & 92 & 6 \\
0.18 & 89 & 15 \\
0.16 & 89 & 13 \\
0.12 & 94 & 5 \\
0.12 & 89 & 11 \\
0.12 & 81 & 14 \\
0.1 & 75 & 6 \\
0.1 & 69 & 25
\end{tabular}

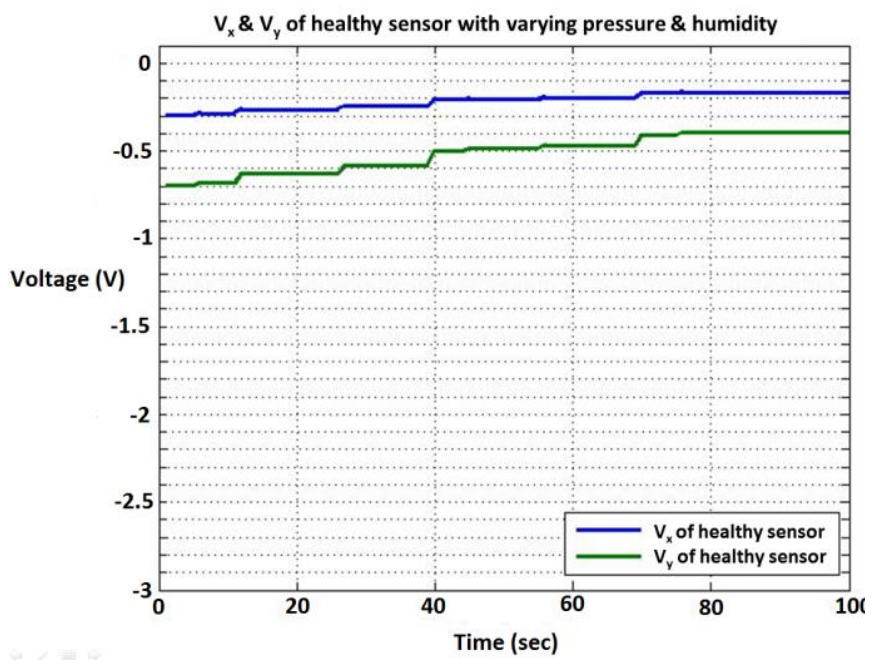

Fig 10: $V_{x}$ and $V_{y}$ for the healthy sensor

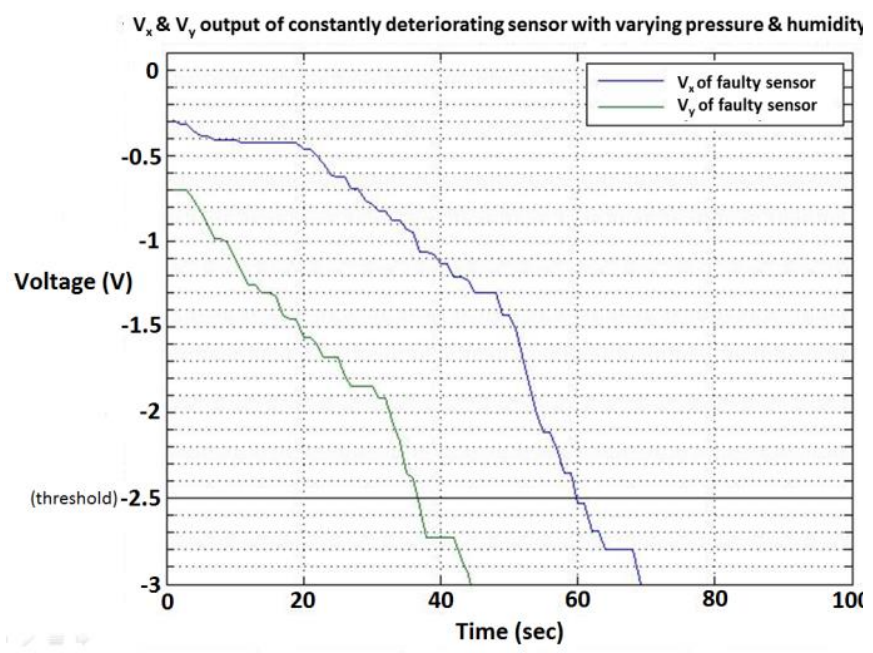

Fig 11: Vx and Vy for the faulty sensor for the same input

The functional flow of the prognostics algorithm is illustrated in Fig 12. This module is dependent on the data generated by the Fault Calibration (FC) module discussed in section V.

During operation of the Fault Calibration, pressure and humidity coefficients are modified when a fault is detected in a sensor. The values of these coefficients are logged. The changes in the values of these coefficients relative to their initial values 
form a metric for the health of the system within the Prognostics Module. Logging these coefficients coefficients is used to predict end of life (EOL) of the system by measuring the rate of decay. The variables used to calculate the decay can be understood with reference to Fig 13.

This algorithm outputs a projection of the time to failure. It also raises a flag when the system moves past a preliminary threshold. This can be used as an early warning system when the sensors are close to total failure. In its current form, it is not equipped to preempt sudden system failures. However, it is suited to the detection of aging or gradual degradation with a good degree of confidence.

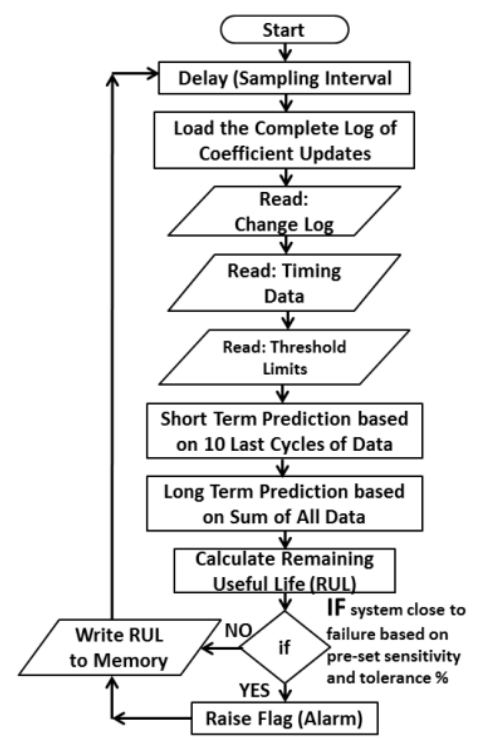

Fig 12: Prognostics Flow Chart. The 4 coefficients of Humidity and Pressure ( $\left.\boldsymbol{\alpha}_{\varphi y} \boldsymbol{\alpha}_{\varphi x} \boldsymbol{\alpha}_{p y} \boldsymbol{\alpha}_{p x}\right)$ are adjusted as they deteriorate. The prognostics algorithm predicts the RUL based on the (rate of) change in the coefficients approaching a complete failure threshold defined for each coefficient individually

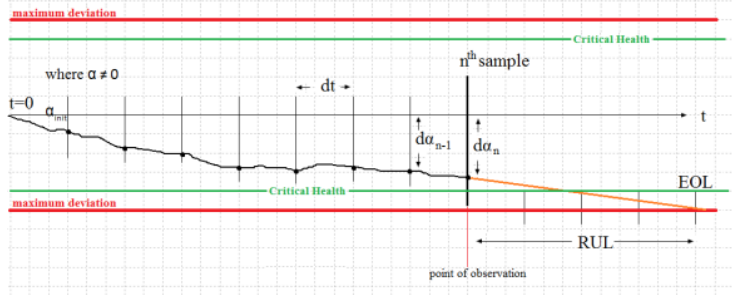

Fig 13: A generic diagram showing the operation of the prognostics system over time. It illustrates observations at fixed intervals and the failure threshold (maximum deviation) the faulty sensor is approaching. The mandate of the Prognostic System requires the determination of RUL (Remaining Useful Life) based on a set of recent observations.

As discussed, the deviation of coefficients from their initial values is used to estimate the deterioration of system health. The RUL value decreases as the system health deteriorates, hence, the net change in recalibrated coefficients relative to their initial values is inversely proportional to the RUL as expressed in (23) \& (24):

$$
\begin{gathered}
R U L \propto \frac{1}{\partial \alpha} \\
R U L \propto\left(\frac{1}{\frac{\partial \alpha_{n}-\partial \alpha_{n-1}}{\partial t}}\right)
\end{gathered}
$$

Where $\partial \alpha_{n}$ is the change in a coefficient relative to an initial value for a healthy sensor at the time of observation $n, \partial \alpha_{n-1}$ is the change in coefficient relative to the initial value in the previous observation. $\partial t$ is the time between observations. Equation (24) can be modified to incorporate observations from any number of previous iterations for increased reliability. The RUL based on the entire length of operation of the sensor can be expressed as (25).

$$
R U L \propto \frac{1}{\frac{\partial \alpha_{n}}{\sum_{1}^{n} \partial t}}
$$

Equation (24) \& (25) can be combined as follows to incorporate both short and long term trends:

$$
R U L \propto \frac{1}{\partial \alpha_{n}} \cdot \frac{1}{k_{1} \frac{\partial \alpha_{n}}{\sum_{1}^{n} \partial t}+k_{2} \frac{\partial \alpha_{n}-\partial \alpha_{n-1}}{\partial t}}
$$

Where $k_{1}$ is the (constant) weight for the short term relative trend and $k_{2}$ is the constant for the long term trend where $\mathrm{k}_{1}$ and $\mathrm{k}_{2}$ can be adjusted as long as $\boldsymbol{k}_{\mathbf{1}}+\boldsymbol{k}_{\mathbf{2}}=\mathbf{1}$

The remaining useful life is directly proportional to the breadth of the threshold available. The smaller the acceptable window of operation, the shorter the RUL is.

$$
R U L \propto \alpha_{e o l}
$$

Equation (26) \& (27) have been combined to form (28) and forms the basis of the prognostics algorithm. For the purposes of the experiment documented in this paper, $k_{1}$ and $k_{2}$ are 0.5 each.

$$
\begin{aligned}
& R U L \\
& =K \cdot \alpha_{e o l} \cdot \frac{1}{\partial \alpha_{n}} \cdot \frac{1}{0.5 \cdot \frac{\partial \alpha_{n}}{\sum_{1}^{n} \partial t}+0.5 \cdot \frac{\partial \alpha_{n}-\partial \alpha_{n-1}}{\partial t}}
\end{aligned}
$$

Where, $K$ is a dynamic constant that is a function of the initial value of the coefficient as well as the previous value of RUL.

\section{A. Tests and results}

We have presented the example of a rapidly deteriorating MFS. The prognostic algorithm has been tested on that particular sensor and the results demonstrate the performance of the prognostics module. It stands to be noted that for the tests presented here, the rate of degradation of the coefficients are abnormally high and serves to demonstrate how the system predicts failure over a short horizon with a tight threshold.

For the purposes of this validation experiment, only one of the humidity coefficients $\left(\alpha_{\phi x}\right)$ has been used. The experiment has concluded that two of the coefficients are more sensitive to failure in humidity sensing, while the other two are more sensitive to failures in pressure transduction. However, as the net mechanical load on the piezoresistors due to pressure is lower than that due to hygroscopic expansion of the polymer beams, only one humidity coefficient was deemed sufficient to 
prognose EOL.

The values of $\alpha_{\phi x}$ stored by the FC were observed every second. The prognostics module runs in 10 second intervals. The failure threshold for $\alpha_{\phi x}$ has been set at -25 volts $/ \%$ RH. It should be noted that while short intervals have been used to demonstrate the modules function, in practice longer intervals (with a relatively higher failure threshold) will be used.

Figure 14 shows observed values of $\alpha_{\phi x}$ (blue line). The prognostics module predicts the RUL every 10 seconds. 4 (out of 7) RUL predictions are presented in Table 5 and in Fig 14 (green lines). As can be seen, the system eventually fails at around the 87 seconds mark.

TABLE 5

predicted RUL calculated by the Prognostics Module over 20 second iterations

\begin{tabular}{|ll|}
\hline Time & Predicted RUL \\
\hline 20 seconds & 728 seconds \\
40 seconds & 180.03 seconds \\
60 seconds & 54.18 seconds \\
80 seconds & 12.72 seconds \\
\hline
\end{tabular}

For this experiment, both the short and long term trends have been configured for equal weight $\left(\mathrm{k}_{1}=\mathrm{k}_{2}=0.5\right)$. The RULs calculated at times 20 and 40 seconds are indicative of the fairly stable state the coefficient is in. At the 60 second mark, the program takes into account the rapid drop in the coefficient value and gives a fairly pessimistic prediction about the EOL of the system. A sudden stabilization of the coefficient can be observed around the 75 and 80 seconds mark. However, the prognostics algorithm takes into account the $\mathrm{K}$ derived from the previous predicted RUL value (at the 70 second mark) and predicts a system failure in 12.72 seconds (at 92.72 seconds). The system actually fails at about the 82 second mark.

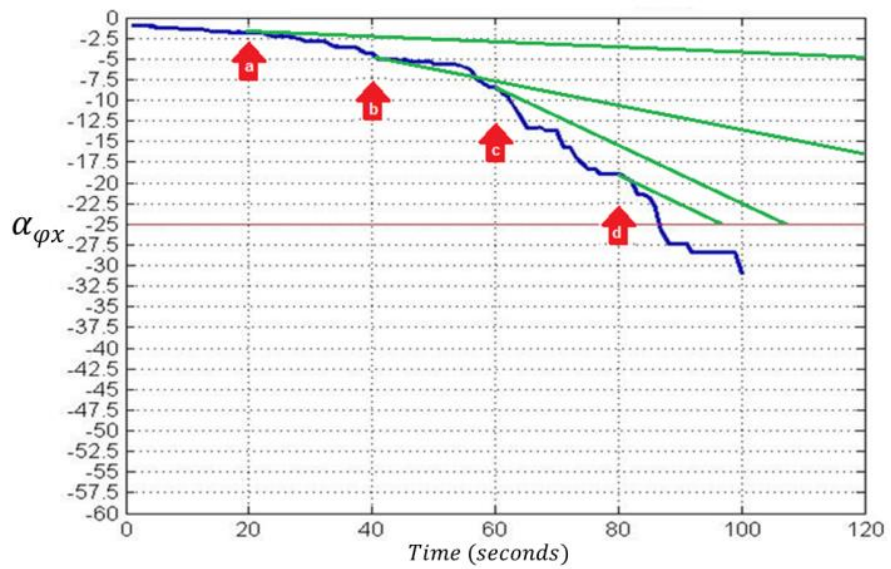

Fig 14: $\alpha_{\phi x}$ observed till failure.(a)System failure predicted in 728 s (b) System failure predicted in 120s (c) System failure predicted in $54.16 s(d)$ System failure predicted in 12.72 seconds, with failure eventually occurring in approx. 8 seconds around the 87 second mark. [Please note: green lines and arrows were superimposed by hand for illustration purposes based on EOL predictions generated by the prognostics module].

This algorithm becomes more precise the longer it runs. Real life deterioration is not expected to be as rapid as demonstrated in this proof of concept. The algorithm was tested over a hundred times for close to a thousand cycles of prognosis per test for very gradual deterioration compared to the example cited. In these tests, the system is able to predict the failure at a long horizon with an average 93\% accuracy. An average accuracy of about $78 \%$ was achieved when the horizon was shortened to 50 cycles prior to failure. Based on these studies, the accuracy becomes better with time and is capable to deliver very good predictions for slow gradual failures over a long period of time.

\section{7. CONCLUSIONS}

This paper presents a mechanism to detect performance degradation in a multifunctional sensor, to calibrate it and to use the data generated by the calibration process in a prognostic algorithm to provide meaningful lifetime estimates for the sensor. The prognostic algorithm is unique in the way it uses changes in behavioral coefficients initiated by the corrective biasing process instead of physical parameters directly from the system under study. This work introduces the novel concept of a low-level prognostics module that can be embedded within a MEMS sensing device with very low processing and memory overhead. The solution has been shown to deliver unprecedented predictive performance for gradually aging systems.

The diagnostic-prognostic module presented in this case study presented a dependable and fault tolerant multi-functional sensor systems well suited to environments requiring high reliability. A prognostic module was developed and it was able to predict the RUL of the multifunctional sensor with approximately $93 \%$ within its defined mandate.

\section{REFERENCES}

[1] B. Larder, "Assessing the benefit of helicopter health and usage monitoring systems," 2003.

[2] J. E. Land, "HUMS-the benefits-past, present and future," in Aerospace Conference, 2001, IEEE Proceedings., 2001, pp. 3083-3094.

[3] E. Mounier and Y. Développement, "MEMS Markets \& Applications," in 2nd workshop on design, control and software implementation for distributed MEMS, 2012.

[4] M. Tariq Jan, N. Hisham Bin Hamid, M. H. Md Khir, K. Ashraf, and M. Shoaib, "Reliability and Fatigue Analysis in Cantilever-Based MEMS Devices Operating in Harsh Environments," Journal of Quality and Reliability Engineering, vol. 2014, 2014. H. S. Newman, J. L. Ebel, D. Judy, and J. Maciel, "Lifetime measurements on a high-reliability RFMEMS contact switch," Microwave and Wireless Components Letters, IEEE, vol. 18, pp. 100-102, 2008.

[6] R. Stefanini, M. Chatras, P. Blondy, and G. M. Rebeiz, "Miniature MEMS switches for RF applications," Microelectromechanical Systems, Journal of, vol. 20, pp. 1324-1335, 2011. 
[7] A. Richardson, H. Van Heeren, and S. Neylon, "Microsystems for structural health monitoring," 2008.

[8] H. Al-Asaad, B. T. Murray, and J. P. Hayes, "Online BIST for embedded systems," IEEE Design \& Test of Computers, vol. 15, pp. 17-24, 1998.

[9] J. Sikorska, M. Hodkiewicz, and L. Ma, "Prognostic modelling options for remaining useful life estimation by industry," Mechanical Systems and Signal Processing, vol. 25, pp. 1803-1836, 2011.

[10] H. Johari, J. S. Yokum, C. Furlong, and R. J. Pryputniewicz, "MEMS sensors for measurements of temperature, pressure, and relative humidity."

[11] Z. Xu, D. Koltsov, A. Richardson, L. Le, and M. Begbie, "Design and simulation of a multi-function MEMS sensor for health and usage monitoring," in Prognostics and Health Management Conference, 2010. PHM'10., 2010, pp. 1-7.

[12] S.-J. Park, J. C. Doll, A. J. Rastegar, and B. L. Pruitt, "Piezoresistive cantilever performance_-part II: optimization," Microelectromechanical Systems, Journal of, vol. 19, pp. 149-161, 2010.

[13] S.-J. Park, J. C. Doll, and B. L. Pruitt, "Piezoresistive cantilever performance_-part I: analytical model for sensitivity," Microelectromechanical Systems, Journal of, vol. 19, pp. 137-148, 2010.

[14] H. Takahashi, N. M. Dung, K. Matsumoto, and I. Shimoyama, "Differential pressure sensor using a piezoresistive cantilever," Journal of micromechanics and microengineering, vol. 22, p. 055015, 2012.

[15] A. A. Mohammed, W. A. Moussa, and E. Lou, "High sensitivity MEMS strain sensor: design and simulation," Sensors, vol. 8, pp. 2642-2661, 2008.

[16] R. Littrell and K. Grosh, "Modeling and characterization of cantilever-based MEMS piezoelectric sensors and actuators," Microelectromechanical Systems, Journal of, vol. 21, pp. 406-413, 2012.

[17] D. Li, T. Li, and D. Zhang, "A monolithic piezoresistive pressure-flow sensor with integrated signal-conditioning circuit," Sensors Journal, IEEE, vol. 11, pp. 2122-2128, 2011.

[18] K. Yamada, M. Nishihara, S. Shimada, M. Tanabe, M. Shimazoe, and Y. Matsuoka, "Nonlinearity of the piezoresistance effect of p-type silicon diffused layers," Electron Devices, IEEE Transactions on, vol. 29, pp. 71-77, 1982.

[19] O. Tufte and E. Stelzer, "Piezoresistive properties of silicon diffused layers," Journal of applied physics, vol. 34, pp. 313-318, 1963.

[20] C. T. Leondes, Mems/Nems:(1) Handbook Techniques and Applications Design Methods,(2)

Fabrication Techniques,(3) Manufacturing Methods,(4) Sensors and Actuators,(5) Medical Applications and MOEMS: Springer, 2007.

[21] F. Battiston, J.-P. Ramseyer, H. Lang, M. Baller, C. Gerber, J. Gimzewski, et al., "A chemical sensor based on a microfabricated cantilever array with simultaneous resonance-frequency and bending readout," Sensors and Actuators B: Chemical, vol. 77, pp. 122-131, 2001.

R. Buchhold, A. Nakladal, G. Gerlach, K. Sahre, K.J. Eichhorn, and M. Müller, "Reduction of mechanical stress in micromachined components caused by humidity-induced volume expansion of polymer layers," Microsystem technologies, vol. 5, pp. 3-12, 1998.

[23] A J Pang \& M Desmulliez, "Modelling and simulation of a silicon micro-diaphragm piezoresistive pressure sensor using Finite Element Analysis (FEA) tools," Proceedings of SPIE - the International Society for Optical Engineering, vol. 4407, pp. 327-336, 2001.

[24] W. D. Pilkey, Formulas for stress, strain, and structural matrices: John Wiley \& Sons, Inc., 1993.

[25] B. Abdelaziz, K. Fouad, and S. Kemouche, "The Effect of Temperature and Doping Level on the Characteristics of Piezoresistive Pressure Sensor," Journal of Sensor Technology, vol. 4, p. 59, 2014.

[26] F. Kerrour, A. Boukabache, and P. Pons, "Modelling of thermal behavior N-doped silicon resistor," Journal of Sensor Technology, vol. 2, p. 132, 2012.

[27] R. Otmani, N. Benmoussa, and B. Benyoucef, "The thermal drift characteristics of piezoresistive pressure sensor," Physics Procedia, vol. 21, pp. 47-52, 2011.

[28] A. A. Mohammed, W. A. Moussa, and E. Lou, "High-performance piezoresistive MEMS strain sensor with low thermal sensitivity," Sensors, vol. 11, pp. 1819-1846, 2011.

[29] Y. Kanda, "A graphical representation of the piezoresistance coefficients in silicon," IEEE Transactions on electron devices, vol. 29, pp. 64-70, 1982.

[30] Y. Kanda, "Piezoresistance effect of silicon," Sensors and Actuators A: Physical, vol. 28, pp. 83-91, 1991. 